\title{
Management of peritonitis in a rural tertiary care institute
}

\author{
Bhatia $S^{1}$, Goel $V^{2}$, Singal $U^{3}$, Verma $S^{4}$ \\ ${ }^{1}$ Dr Sanjiv Bhatia, Associate Professor, Department of General Surgery, ${ }^{2}$ Dr. Vijay Kumar Goel, Associate Professor, \\ Department of General Surgery, ${ }^{3}$ Dr. Umang Singal, Senior Resident, Department of General Surgery, above ${ }^{1,2,3}$ authors are \\ affiliated with Hind Institute Of Medical Sciences, Safedabad, Barabanki, Uttarpradesh, ${ }^{4}$ Dr. Satyajeet Verma, Professor, \\ Department of General Surgery, Government Medical College, Azamgarh, Uttar Pradesh, India.
}

Address for Correspondence: Dr Vijay Kumar Goel, Associate Professor, Department of General Surgery, Hind Institute Of Medical Sciences, Safedabad, Barabanki, Uttarpradesh, India, Email: vijaygoe11981@gmail.com

\begin{abstract}
Introduction: Peritonitis is defined as the inflammation of the peritoneal cavity. Peritonitis due to perforation of gastro intestinal hollow viscus is the common surgical emergency in India and the spectrum of disease is different from that found in the western world. Material and Methods: This study was done to know the various trends of management in peritonitis cases in Barabanki district of Uttar Pradesh state of India. In this study of 200 cases of peritonitis coming to Hind Institute of Medical Sciences, Barabanki, Uttar Pradesh were screened on the basis of lab investigations, X-rays (Chest \& abdomen), ultrasonography, and abdominal aspiration when required. Results: Majority of perforation peritonitis patient presented to emergency with dyselectrolemia, followed by septicemia, ultra-sonography showed free fluid and four quadrant aspiration s/o nature of peritoneal cavity fluid. Patients managed with initial resuscitation and surgery to correct the underlying pathology. Conclusion: Closure of perforation with omental patching done in stomach and duodenal perforation with peritoneal toilet. Omental patching is the commonest surgical procedure done in the peptic perforations.
\end{abstract}

Keywords: Peritonitis, Omental Patching, Peritoneal toilet, Perforation.

\section{Introduction}

Peritonitis is defined as inflammation of the serosal membrane that lines the abdominal cavity and the organs contained therein. Peritonitis due to hollow viscus perforation continues to be the one of the most common surgical emergencies confronting surgeons. Peritonitis is a common complication of peritoneal dialysis. Peritonitis is associated with significant morbidity, catheter loss, and transfer to hemodialysis, transient loss of ultrafiltration, possible permanent membrane damage and occasionally death $[1,2]$.

Peritonitis was a frequently associated side effect that hindered the acceptance of chronic peritoneal dialysis until an improved access catheter was developed by Henry Tenckhoff in 1968. This catheter significantly decreased the incidence of peritonitis, but initial of patients undergoing continuous ambulatory peritoneal dialysis (CAPD) with this catheter indicated peritonitis rates of more than six episodes per patient per year [3]. Peritonitis due to perforation of gastro intestinal hollow viscus is the common surgical emergency in India and the

Manuscript Received: $14^{\text {th }}$ July 2017

Reviewed: $24^{\text {th }}$ July 2017

Author Corrected: $3^{\text {rd }}$ August 2017

Accepted for Publication: $10^{\text {th }}$ August 2017 spectrum of disease is different from that found in the western world. The advent of proton pump inhibitors and helicobacter pylori eradications in the management of chronic peptic ulcer disease has reduced the operative treatment of this condition and its complications. But yet perforated duodenal ulcer remains a major life threatening complication of chronic peptic ulcer disease. Despite advances in surgical techniques, antimicrobial therapy and intensive care support, management of peritonitis continues to be highly demanding, difficult and complex [4].

Peritonitis is frequently encountered surgical emergency in any hospital with high morbidity and mortality, which continues to be a matter of great concern to the surgeons, particularly in a tropical country like India. At the time of presentation, general condition of the patient was usually very much deteriorated and deserves skillful emergency surgical management.

\section{Material and Methods}

It was a cross-sectional study. 200 cases with provisional diagnosis of peritonitis which came to the surgical 
Original Research Article

emergency of Hind Institute of Medical Sciences, Barabanki, Uttar Pradesh were reviewed.

The various methods of management affecting the outcome were studied. Provisional diagnosis was made from the history and clinical examination of the patient. To clinch the final diagnosis, various investigations like $\mathrm{Hb}$, TLC, DLC, blood urea, blood sugar, serum creatinine, complete microscopic examination and culture sensitivity of fluid extracted by paracentesis. Radiological investigations including $\mathrm{x}$-ray chest (PA view) standing including both domes of diaphragm, x-ray abdomen standing position, including both domes of diaphragm, ultrasound abdomen, CT abdomen (whenever indicated) followed by respective treatment.

\section{Result}

A total of 200 patients with provisional diagnosis of peritonitis were taken up for study. Most common cause of peritonitis was perforation (total 140 cases). The most common anatomical site for perforation was terminal ileum (68 cases); the next common site was stomach (44 cases) followed by duodenum (20 cases) and jejunum (4 cases), colon (4 cases).

Anaemia was present in $12 \%$ of cases. $22 \%$ case presented with septicaemia (TLC $>12000 / \mathrm{mm} 3$ ).

Electrolyte imbalance was seen in $40 \%$ case. Four quadrant aspiration analysis which showed that fecal matter was in aspirate in $36 \%$ cases. Clear fluid was present in $22 \%$ cases. Bilious fluid was present in $12 \%$ cases. Hemorrhagic fluid was present in $4 \%$ cases. Pus was present in $4 \%$ cases; no aspiration fluid was seen in $22 \%$ cases.

Suturing of the perforation was the most common (64 cases) surgical procedure done, omental patching with suturing in 64 cases, resection and anastomosis in the 32 cases. Appendicectomy was done in 16 cases. Ileostomy was done in 28 cases. Cecopexy was done in 8 cases with provisional diagnosis of peritonitis. In 8 cases only peritoneal lavage was done and drains were inserted in not identified or sealed perforation.

Fever was the most common post-operative complication $(90 \%)$, the next common post-operative complication was paralytic ileus (70\%), and superficial wound infection was present in $50 \%$ cases. Other post-operative complications were anaemia/hypoproteinemia (20\%), burst abdomen $(2 \%)$ and chest infection (10\%). Overall mortality was $12 \%$ in this study. Mortality in ileal perforation was $17.6 \%$.

\section{Discussion}

The present study was planned to study the aids to diagnosis and management of peritonitis. In this study the most common cause of peritonitis is perforation peritonitis, various studies favour that perforation is the main cause of peritonitis.

In this study the most common site of perforation was terminal ileum (up to $30 \mathrm{cms}$ proximal to ileocecal junction) present in 68 cases out of 140 cases, next most common site was stomach (44 out of 140 cases ), other sites were $1^{\text {st }}$ part of duodenum (20 cases), jejunum (4 cases), colon (4 cases). Similar observation were found by study of Agrawal $\mathrm{N}$ et al [5]. X-ray chest PA view including both domes of diaphragm in upright position is a very useful investigation to diagnose the perforation of hollow viscus by detecting pneumo-peritoneum.

In this present study X-ray abdomen was done in 200 cases, pneumo-peritoneum was detected in all cases of perforation peritonitis (140 out of 200 cases) and multiple air-fluid levels with pneumo-peritoneum was the finding in 8 cases. This study is well comparable with the study of Sofic et al [6] (x-ray abdomen showed free air in the abdominal cavity in $80 \%$ cases of gastro-intestinal perforation), whereas Dickson JAS et al [7] films in patients of terminal ileum perforation, and Aston $\mathrm{NO}$ et al [3] reported free peritoneal gas in $25 \%$ of abdominal $x$-ray films in patients of perforation.

In this study ultrasound examination of abdomen was done in 200 cases. The most common ultra-sonographic findings were free fluid in the peritoneal cavity and dilated gut loops with sluggish or absent peristalsis $(92 \%$ cases), pneumo-peritoneum was detected in $100 \%$ cases of perforation.

Findings of ultrasonography in this study are well supported by the studies at Sofic et al [6] (free fluid was detected in $90 \%$ cases).

In our study CECT of the abdomen was done only in two cases. Sofic et al [6] had observed that CT was more sensitive to the combination of liquid and minimal amount of free air which was undetectable with ultrasound and Xray.

In the present study management was mainly surgical. Exploratory laparotomy was done in all cases after 3 to 4 hours of initial resuscitation.

Pre-operative resuscitation included I.V. fluids with electrolytes, Ryle's tube aspiration and Foley's 
catheterization, maintenance of input-output balance, blood transfusion, I.V. antibiotics against Gram-positive, Gram-negative and anaerobes, Monitoring of temperature, pulse and blood pressure regularly, pre-operative intra peritoneal abdominal drain insertion to reduce toxemia, was inserted in two cases who were severely toxic and abdomen was over distended causing respiratory distress. Pneumo-peritoneum and signs of peritonitis were observed in all cases of perforation.

Foul smelling clear fluid with or without flakes in the peritoneal cavity was observed in 44 cases; foul smelling bilious fluid with or without flakes was observed in 24 cases; foul smelling fecal matter, with or without flakes were observed in 72 cases, hemorrhagic fluid was observed in 8 cases of trauma.

In 68 cases, terminal ileal perforation (upto $30 \mathrm{~cm}$ proximal to the ileoceacal junction on anti-mesentric border) was observed; in 44 cases gastric perforation was observed. In all the cases of gastric perforation, perforation was single and present either in the pyloric region or on the anterior surface of stomach; in 20 cases perforation was observed on the anterior wall of the $1^{\text {st }}$ part of the duodenum; in 4 cases perforation was observed on the anti mesenteric border of the jejunum; colon perforation was observed in 4 cases.

The aims of surgical intervention are twofold: to drain the pus \& bowel contents from peritoneal cavity and to prevent further contamination.

Minimum required operative procedure was performed. In all the cases of peptic perforation, the edges were excised $\&$ margins freshened and perforation was closed in single layer by applying non absorbable sutures. Pedicled omental patch was also applied in almost all the cases. Meticulous peritoneal toileting was done with normal saline. Abdominal drains were inserted in pelvic cavity and morrison's pouch.

In enteric perforation, simple closure of perforations was done with atraumatic needle in two layer/ single after freshening of margins. As the patients were of poor surgical risk and they tolerated minimum anaesthesia, simple closure has the advantage of being quick and easy. An alternative procedure like resection and ileo-ileal anastomosis were also done in some cases.

In some cases of enteric perforation where the gut was not healthy enough or with multiple perforations or there was excessive soiling, exteriorization of gut was done (ileostomy). Postoperatively patients were given I/V fluids, Ryle's tube aspiration, blood transfusions, antibiotics such as $3^{\text {rd }}$ generation cephalosporins \& metronidazole for anaerobic organisms. This treatment was recommended by most of the previous authors. Antitubercular drugs were given to all patients with tuberculous peritonitis.

Aston NO et al [8] and Sweetman R et al [9] had also recommended resection of the segment of the ileum as a treatment of choice in case of tubercular peritonitis.

Gupta S et al [10] \& other authors have also recommended the omental patching in gastro-duodenal perforations.

Gupta SK et al [11] had also recommended the primary closure of the traumatic perforations. Townsend $\mathrm{MC}$ et al [12] had also recommended the primary closure of traumatic perforations. KIM JP et al [13] recommended resection of small bowel in multiple typhoid perforations of the terminal ileum; he also recommended exteriorization of small bowel in very sick patients.

In this study fever was the most common post-operative complication which was presented in $90 \%$ of the patients.

$2^{\text {nd }}$ most common postoperative complication was paralytic ileus (for $>2$ days) which was presented in $70 \%$ of the patients. Other postoperative complications were superficial wound infections (50\%), anaemia/ hypoproteinemia (20\%), burst abdomen $(2 \%)$, chest infections $(10 \%)$ and anastomotic leaks $(0 \%)$.

Whereas in the study of Gupta SK et al [11] common morbidity encountered was chest infection (39 cases), wound infection (12 cases), biliary leak ( 8 cases), intraabdominal abscesses (6 cases), burst abdomen (6 cases), renal failure ( 2 cases), DIC ( 4 cases), jaundice and upper gastro-intestinal bleeding ( 1 case each).

The overall mortality in the present study is $12 \%$. The causes of mortality in the present series are very poor general condition of the patient at the time of admission, anaemia, toxemia, dehydration and delayed reporting of the patients.

\section{Conclusion}

Laparotomy \& closure of the perforation is still the commonest surgical procedure done in ileal \& jejunal perforations followed by thorough peritoneal toilet with normal saline. Closure of perforation with omental patching done in stomach and duodenal perforation with peritoneal toilet that is omental patching, the commonest surgical procedure done in the peptic perforations. 
Peritoneal toilet \& lavage with normal saline is the essential component of all the surgical procedures in perforation peritonitis.

Resection \& anastomosis \& ileostomy are the surgical procedures done in multiple ileal perforation and /or in case of unhealthy, edematous bowel wall.

Conflict of interest: None declared.

Funding: Nil, Permission from IRB: Yes

\section{References}

1. Holley JL,PrainoBM.Complications of peritoneal dialysis: Diagnosis and treatment. Semin Dial.1990;3:245.

2. Burke CM,BrierME,Golper TA. Outcomes of single organism peritonitis in peritoneal dialysis: gram negatives versus gram positives in the Network 9 Peritonitis Study. Kidney Int.1997;52:524

3. Rubin J, Rogers WA, Taylor HM, Everett ED, Prowant BF, Fruto LV, Nolph KD. Peritonitis during continuous ambulatory peritoneal dialysis. Ann Intern Med. 1980 Jan; 92 (1):7-13.

4. Hainaux B, Agneessens E, Bertinotti R, De Maertelaer V, Rubesova E, Capelluto E, Moschopoulos C. Accuracy of MDCT in predicting site of gastrointestinal tract perforation. AJR Am J Roentgenol. 2006 Nov;187 (5): 1179-83.

5. Agarwal N, Saha S, Srivastava A, Chumber S, Dhar A, Garg S. Peritonitis: 10 years' experience in a single surgical unit. Trop Gastroenterol. 2007 Jul-Sep; 28 (3): 117-20.
6. Sofic A, Besilic S, Linceder L, Vrcic D. Early radiological diagnostics of gastrointestinal perforation. Radiol Oncol. 2006; 40 (2): 67-72.

7. Dickson ja, cole gj. Perforation of the terminal ileum. A review of 38 cases. Br J Surg. 1964 Dec;51:893-7.

8. Aston NO, de Costa AM. Tuberculous perforation of the small bowel. Postgrad Med J. 1985 Mar;61(713): 251-2.

9. SWEETMAN WR, WISE RA. Acute perforated tuberculous enteritis: surgical treatment. Ann Surg. 1959 Jan; 149 (1):143-8.

10. Gupta S, Kaushik R, Sharma R, Attri A. The management of large perforations of duodenal ulcers. BMC Surg. 2005 Jun 25;5:15.

11. Gupta SK, Gupta R, Singh G, Gupta S. Perforation Peitonitis: A Two Year Experience. JK Science. 2010; 12 (3) : 141-4.

12. Townsend MC, Pelias ME. A technique for rapid closure of traumatic small intestinal perforations without resection. Am J Surg. 1992; 164(2): 171 -2.

13. Kim JP, Oh SK, Jarrett F. Management of ileal perforation due to typhoid fever. Ann Surg. 1975 Jan; 181 (1): 88-91.

14. Jain BK, Arora H, Srivastava UK, Mohanty D, Garg PK. Insight into the management of non-traumatic perforation of the small intestine. J Infect Dev Ctries. 2010 Oct 28;4(10):650-4.

\section{How to cite this article?}

Bhatia S, Goel V, Singal U, Verma S. Management of peritonitis in a rural tertiary care institute. Int J surg Orthopedics. 2017;3(3):72-75.doi:10.17511/ijoso.2017.i03.04. 